\title{
Non-uniform bounds for the dermal patch problem via Stein's method
}

\author{
Kamonrat Kamjornkittikoon, Kritsana Neammanee, Nattakarn Chaidee* \\ Department of Mathematics and Computer Science, Faculty of Science, Chulalongkorn University, \\ Bangkok 10330 Thailand
}

*Corresponding author, e-mail: nattakarn.c@chula.ac.th

\begin{abstract}
The number of doses in a dermal patch under the standard protocol of drug administration is analysed via a mathematical model. Goldstein and Zhang gave a uniform Berry-Esseen bound for the number of receptors active at the terminal time by using a version of Stein's method for bounded monotone size-biased couplings. In this paper, we generalize their work to the case of a non-uniform bound.
\end{abstract}

KEYWORDS: bounded monotone size biased couplings, clubbed binomial, uniform and non-uniform bound

MSC2010: 60F05 60E50 62P10

\section{INTRODUCTION}

A medicated adhesive patch which delivers the medication through the skin is called a dermal patch. In pharmaceutical studies, the dermal patch method is a way to measure the amount of pesticide residue directly deposited on the skin ${ }^{1}$. This method is also developed in exposure studies using video imaging techniques and in lymphocyte transformation testing for quantifying metal hypersensitivity ${ }^{2,3}$. A study in the pharmaceutical industry has recognized the potential of using the dermal patch ${ }^{4,5}$.

In this work, we consider how to check the patches before going to market and the effects of dermal patches designed to activate targeted receptors. This system includes inactive receptors which are activated if they receive a dose of medicine released from the dermal patch and deactivated if they receive it once again. The doses are increased progressively for a limited number of days as follows: for the number of receptors $n$, on each day $r=1, \ldots, n$ of the study, there are $r$ randomly selected receptors for which each receives one dose of medicine from the patch. We focus on the number of receptors active at the terminal time $n$. We can simulate the problem using a mathematical model by the following argument.

Let $n \in \mathbb{N}$ be fixed and

$$
\mathbf{X}=\left\{X_{r k} \mid r=0,1, \ldots, n ; k=1, \ldots, n\right\}
$$

be a collection of Bernoulli variables such that for

$$
\begin{aligned}
& r \geqslant 1, \\
& X_{r k}=\left\{\begin{array}{ll}
1, & \text { the status of receptor } k \text { is } \\
0, & \text { otherwise. }
\end{array} \text { changed on day } r,\right.
\end{aligned}
$$

The initial state of the receptor is given deterministically by $\left\{X_{0 k} \mid k=1, \ldots, n\right\}$, which will be taken to be the state zero, that is, all receptors are inactive, unless specifically stated otherwise. At stage $r$, the receptors are chosen uniformly to have their status changed and the stages are independent of each other. Let

$$
X_{k}=\left(\sum_{r=0}^{n} X_{r k}\right) \bmod 2, \quad W_{n}=\sum_{k=1}^{n} X_{k},
$$

where the random variable $X_{k}$ is the indicator that receptor $k$ is active at the terminal time and the random variable $W_{n}$ is the number of receptors active at the terminal time. The mean and the variance of $W_{n}$ are given by ${ }^{4}$

$$
\begin{aligned}
\mu & =E\left(W_{n}\right)=\frac{n}{2}\left(1-\prod_{s=1}^{n} \lambda_{n, 1, s}\right), \\
\sigma^{2} & =\operatorname{Var}\left(W_{n}\right) \\
& =\frac{n}{4}\left(1-\prod_{s=1}^{n} \lambda_{n, 2, s}\right)+\frac{n^{2}}{4}\left(\prod_{s=1}^{n} \lambda_{n, 2, s}-\prod_{s=1}^{n} \lambda_{n, 1, s}^{2}\right),
\end{aligned}
$$

where

$$
\lambda_{n, 1, s}=1-\frac{2 s}{n}, \quad \lambda_{n, 2, s}=1-\frac{4 s}{n}+\frac{4 s(s-1)}{n(n-1)}
$$


for $s=1, \ldots, n$.

Because, in the case of even number $n$,

$$
\prod_{s=1}^{n} \lambda_{n, 1, s}=\prod_{s=1}^{n} \lambda_{n, 1, s}^{2}=0
$$

we obtain $\mu=\frac{1}{2} n$ and

$$
\sigma^{2}=\frac{n}{4}\left(1-\prod_{s=1}^{n} \lambda_{n, 2, s}\right)+\frac{n^{2}}{4}\left(\prod_{s=1}^{n} \lambda_{n, 2, s}\right) .
$$

Goldstein and Zhang ${ }^{6}$ showed that

$$
-\frac{\mathrm{e}^{-n}}{n-1} \leqslant \prod_{s=1}^{n} \lambda_{n, 2, s} \leqslant \frac{\mathrm{e}^{-n}}{n-1}
$$

which implies

$$
\frac{n}{4}\left(1-\mathrm{e}^{-n}\right) \leqslant \sigma^{2} \leqslant \frac{n}{4}\left(1+\mathrm{e}^{-n}\right) .
$$

In addition, the distribution of $W_{n}$ can be approximated by the standard normal distribution function $\Phi^{6}$. Their technique is Stein's method for bounded monotone size-biased couplings and the result is the following.

Theorem 1 (Ref. 6) For all even numbers $n$ such that $n \geqslant 6$,

$$
\begin{aligned}
& \sup _{z \in \mathbb{R}}\left|P\left(\frac{W_{n}-n / 2}{\sigma} \leqslant z\right)-\Phi(z)\right| \\
& \leqslant \frac{n}{2 \sigma^{2}}\left(\frac{1}{2 \sqrt{n}}+\frac{1}{2 n}+\frac{1}{3} \mathrm{e}^{-n / 2}\right)+1.64 \frac{n}{\sigma^{3}}+\frac{2}{\sigma} .
\end{aligned}
$$

In the other case, for $n=2 m+1$, an odd number, Goldstein and Zhang ${ }^{6}$ constructed a symmetrical random variable $V$ which is close to $W_{n}$ and normalized $W_{n}$ by the mean and the variance of $V$ given by

$$
\begin{aligned}
\mu_{V} & =\frac{n}{2}\left(1-\prod_{r=1}^{n} \bar{\lambda}_{n, 1, r}\right)=\frac{n}{2}, \\
\sigma_{V}^{2} & =\frac{n}{4}\left(1-\prod_{r=1}^{n} \bar{\lambda}_{n, 2, r}\right)+\frac{n^{2}}{4}\left(\prod_{r=1}^{n} \bar{\lambda}_{n, 2, r}\right),
\end{aligned}
$$

where

$$
\bar{\lambda}_{n, b, r}= \begin{cases}\frac{1}{2}\left(\lambda_{n, b, m}+\lambda_{n, b, m+1}\right), & r \in\{m, m+1\}, \\ \lambda_{n, b, r}, & \text { otherwise }\end{cases}
$$

for $b=1,2$. Since they proved that

$$
-\mathrm{e}^{-n} \leqslant \prod_{\substack{r=1 \\ r \neq m}}^{n} \bar{\lambda}_{n, 2, r} \leqslant \mathrm{e}^{-n}
$$

we obtain

$$
\frac{n}{4}-\frac{n-1}{4} \mathrm{e}^{-n} \leqslant \sigma_{V}^{2} \leqslant \frac{n}{4}+\frac{n-1}{4} \mathrm{e}^{-n}
$$

and obtain the following result.

Theorem 2 (Ref. 6) For all odd numbers $n \geqslant 7$,

$$
\begin{aligned}
& \sup _{z \in \mathbb{R}} \mid P\left(\frac{W_{n}-n / 2}{\sigma_{V}} \leqslant z\right)-\Phi(z) \mid \\
& \leqslant \frac{n}{2 \sigma_{V}^{2}}\left(\frac{1}{\sqrt{n}}+\frac{1}{2 \sqrt{2}} \mathrm{e}^{-n / 4}\right)+1.64 \frac{n}{\sigma_{V}^{3}} \\
&+\frac{2}{\sigma_{V}}\left(1+\frac{1}{\sqrt{2 \pi}}\right) .
\end{aligned}
$$

An approximation of the distribution of $W_{n}$ by the clubbed binomial distribution via Stein's method was suggested in Goldstein and Xia ${ }^{7}$. We say that the random variable $C_{n}$ has the clubbed binomial distribution if $C_{n}$ has the following distribution. If $n \bmod 4 \in\{0,3\}$,

$$
P\left(C_{n}=i\right)= \begin{cases}\left(\begin{array}{l}
n \\
i
\end{array}\right)\left(\frac{1}{2}\right)^{n-1}, & i \in \mathbb{E}_{n}, \\
0, & \text { otherwise, }\end{cases}
$$

and if $n \bmod 4 \in\{1,2\}$,

$$
P\left(C_{n}=i\right)= \begin{cases}\left(\begin{array}{l}
n \\
i
\end{array}\right)\left(\frac{1}{2}\right)^{n-1}, & i \in \mathbb{O}_{n}, \\
0, & \text { otherwise, }\end{cases}
$$

where $\mathbb{E}_{n}\left(\mathbb{O}_{n}\right)$ is the set of even (odd) numbers in $\{0,1, \ldots, n\}$. The result is

$$
\sup _{A \subseteq \mathbb{Z}}\left|P\left(W_{n} \in A\right)-P\left(C_{n} \in A\right)\right| \leqslant 2.7314 \sqrt{n} \mathrm{e}^{-(n+1) / 3} \text {. }
$$

In this work, we generalize Theorem 1 and Theorem 2 to the case of a non-uniform exponential bound using the non-uniform bound in the normal approximation for a binomial random variable and the result of the clubbed binomial approximation for $W_{n}$. These are our results.

Theorem 3 For all even numbers $n \geqslant 6$ and for all real numbers $z$ such that $0<|z| \leqslant \sqrt{n}$,

$$
\left|P\left(\frac{W_{n}-n / 2}{\sigma} \leqslant z\right)-\Phi(z)\right| \leqslant \varepsilon(n, z),
$$

where

$$
\begin{aligned}
\varepsilon(n, z)=2.7314 \sqrt{n} \mathrm{e}^{-(n+1) / 12-\left(z^{2}+1\right) / 4} & \\
+\frac{1}{\sqrt{n-1}}\left[9.6414 \mathrm{e}^{-z^{2} / 2}\right. & +21.9638 \mathrm{e}^{-z^{2} / 12} \\
& \left.+17.1396 \mathrm{e}^{-z^{2} / 16}\right] .
\end{aligned}
$$


Theorem 4 For all odd numbers $n \geqslant 7$ and for all real numbers $z$ such that $0<|z| \leqslant \sqrt{n}$,

$$
\left|P\left(\frac{W_{n}-n / 2}{\sigma_{V}} \leqslant z\right)-\Phi(z)\right| \leqslant \varepsilon(n, z) .
$$

Remark 1 Note that $0 \leqslant W_{n} \leqslant n$ and $\sigma^{2} \approx \frac{1}{4} n$ (exponentially). Then $P\left(\left(W_{n}-n / 2\right) / \sigma \leqslant z\right)=1$ for all $z>\sqrt{n}$ and $P\left(\left(W_{n}-n / 2\right) / \sigma \leqslant z\right)=0$ for all $z<-\sqrt{n}$. Hence it is reasonable to consider the bounds when $|z| \leqslant \sqrt{n}$. In addition, by (1) and (2), we see that $0.249 n \leqslant \sigma^{2} \leqslant 0.251 n$ for $n \geqslant 6$ and $0.249 n \leqslant \sigma_{V}^{2} \leqslant 0.251 n$ for $n \geqslant 7$, respectively.

To prove our main results, we use the normal approximation for independent bounded random variables.

\section{NON-UNIFORM EXPONENTIAL BOUNDS FOR NORMAL APPROXIMATION TO INDEPENDENT BOUNDED RANDOM VARIABLES}

Let $Y_{1}, Y_{2}, \ldots, Y_{n}$ be independent and not necessary identically distributed random variables with zero means and finite variances. Let

$$
S_{n}=\sum_{i=1}^{n} Y_{i}
$$

and assume that $\operatorname{Var}\left(S_{n}\right)=1$.

Under the assumption of the finiteness of the third moment, we have the uniform Berry-Esseen theorem

$$
\left|P\left(S_{n} \leqslant z\right)-\Phi(z)\right| \leqslant C_{0} \sum_{i=1}^{n} E\left|Y_{i}\right|^{3}
$$

and the non-uniform Berry-Esseen theorem

$$
\left|P\left(S_{n} \leqslant z\right)-\Phi(z)\right| \leqslant \frac{C_{1}}{1+|z|^{3}} \sum_{i=1}^{n} E\left|Y_{i}\right|^{3},
$$

where $C_{0}$ and $C_{1}$ are absolute constants. If the $Y_{i}$ are identically distributed, then both inequalities shown above can be found in the original works of Esseen ${ }^{8}$ and Nagaev ${ }^{9}$, respectively. The upper estimate of the constant $C_{0}$ has been much studied $^{10-12}$. The best estimate $C_{0}<0.4748$ was given in Ref. 13. The result in the case that $Y_{i}^{\prime}$ 's are nonidentically distributed was generalized in Ref. 14 and the best estimate $C_{0}=0.5600$ was obtained in Ref. 15. For the non-uniform version, the best constant $C_{1}$ was calculated to be 31.395 for nonidentically distributed random variables ${ }^{16}$. Chen and Shao ${ }^{17}$ gave the non-uniform bound without assuming the existence of the third moment. In the case of bounded random variables, that is, $\left|Y_{i}\right| \leqslant \delta$ for $i=1, \ldots, n$, they obtained the uniform bound as $^{18}$

$$
\left|P\left(S_{n} \leqslant z\right)-\Phi(z)\right| \leqslant 3.3 \delta .
$$

Chaidee $^{19}$ used the idea of Chen and Shao ${ }^{18}$ to prove a non-uniform exponential bound for independent bounded random variables

$$
\left|P\left(S_{n} \leqslant z\right)-\Phi(z)\right| \leqslant C_{\delta} \mathrm{e}^{-|z| / 2} \delta,
$$

where $C_{\delta}=4.45+2.21 \exp \left(2 \delta+\delta^{-2}\left(\mathrm{e}^{2 \delta}-1-\right.\right.$ $2 \delta)$ ). Furthermore, a non-uniform exponential bound in terms of $\mathrm{e}^{-z^{2} / 2}$ with unknown constant was proved ${ }^{20}$. In this section, we give a nonuniform exponential Berry-Esseen bound for independent bounded random variables in terms of $\mathrm{e}^{-z^{2} / k}$ equipped with some known constants by using ideas from Chen and Shao ${ }^{18}$ and the technique from Ref. 19 which uses Stein's method.

Stein ${ }^{21}$ introduced the method to find a BerryEsseen bound which does not use the Fourier transform and depends on an elementary differential equation. First, we consider Stein's equation for the normal distribution:

$$
f^{\prime}(w)-w f(w)=\mathbb{I}(w \leqslant z)-\Phi(z),
$$

where $\mathbb{I}$ is the indicator function. This linear differential equation has the unique solution,

$$
f_{z}(w)= \begin{cases}\tilde{w} \Phi(w)[1-\Phi(z)], & w \leqslant z \\ \tilde{w} \Phi(z)[1-\Phi(w)], & w>z .\end{cases}
$$

where $\tilde{w}=\sqrt{2 \pi} \mathrm{e}^{w^{2} / 2}$. Then,

$$
f_{z}^{\prime}(w)= \begin{cases}{[1-\Phi(z)][1+w \tilde{w} \Phi(w)],} & w<z, \\ \Phi(z)[w \tilde{w}(1-\Phi(w))-1], & w>z .\end{cases}
$$

For any random variable $W$, by (5), we note that

$$
E\left(f_{z}^{\prime}(W)\right)-E\left(W f_{z}(W)\right)=P(W \leqslant z)-\Phi(z)
$$

which implies that we can find a bound of $E\left(f_{z}^{\prime}(W)\right)-E\left(W f_{z}(W)\right)$ instead of $P(W \leqslant z)-\Phi(z)$. To bound $\left|E\left(f_{z}^{\prime}(W)\right)-E\left(W f_{z}(W)\right)\right|$, we rewrite $E\left(W f_{z}(W)\right)$ in a suitable form by using the sizebiased coupling approach ${ }^{22,23}$.

For a nonnegative random variable $W$ with mean $\mu$, we say $W^{s}$ has the $W$-size biased distribution if for all functions $f$ for which $E(W f(W))$ exists,

$$
E(W f(W))=\mu E f\left(W^{s}\right) .
$$

We need Lemma 1 to obtain a non-uniform bound in Theorem 5 and Lemma 2 to give bounds in our main theorems. 
Lemma 1 Let $W$ be a nonnegative random variable with mean $\mu$ and variance $\sigma^{2}$, both finite and positive, and let $W^{s}$ have the $W$-size biased distribution and $U=(W-\mu) / \sigma$. If $W \leqslant W^{s} \leqslant W+B$ with probability 1 , for some $B>0$, then for any $k>0$ such that $k B \leqslant$ $\sigma$,

$$
E \mathrm{e}^{k U} \leqslant \mathrm{e}^{\mu k^{2} B / 2 \sigma^{2}+2 \mu k^{3} B^{2} / 3 \sigma^{3}} .
$$

Proof: Let $\tilde{U}=\left(W^{s}-\mu\right) / \sigma$. Since $W^{s}$ has the $W$-size biased distribution,

$$
E(U f(U))=\frac{\mu}{\sigma} E(f(\tilde{U})-f(U))
$$

for all functions $f$ for which these expectations exist $^{22}$. Let $f: \mathbb{R} \rightarrow \mathbb{R}$ and $h:(0, \infty) \rightarrow \mathbb{R}$ be defined by $f(u)=\mathrm{e}^{k u}$ and $h(t)=E \mathrm{e}^{t U}$. Note that

$$
\tilde{U}-U=\frac{W^{s}-W}{\sigma} \leqslant \frac{B}{\sigma} .
$$

Thus by (6) and (7), we obtain

$$
\begin{aligned}
h^{\prime}(k) & =E\left(U \mathrm{e}^{k U}\right) \\
& =\frac{\mu}{\sigma} E[f(\tilde{U})-f(U)] \\
& =\frac{\mu}{\sigma} E \int_{0}^{\tilde{U}-U} f^{\prime}(U+t) \mathrm{d} t \\
& =\frac{\mu}{\sigma} E \int_{0}^{\tilde{U}-U} k \mathrm{e}^{k(U+t)} \mathrm{d} t \\
& \leqslant \frac{\mu k}{\sigma} E \int_{0}^{B / \sigma} \mathrm{e}^{k(U+B / \sigma)} \mathrm{d} t \\
& =\frac{\mu k B}{\sigma^{2}} \mathrm{e}^{k B / \sigma} E \mathrm{e}^{k U} \\
& \leqslant \frac{\mu k B}{\sigma^{2}} E \mathrm{e}^{k U}+\frac{\mu k B}{\sigma^{2}} E \mathrm{e}^{k U}\left|\mathrm{e}^{k B / \sigma}-1\right| \\
& \leqslant \frac{\mu k B}{\sigma^{2}} E \mathrm{e}^{k U}+\frac{2 \mu k^{2} B^{2}}{\sigma^{3}} E \mathrm{e}^{k U}
\end{aligned}
$$

where the last inequality holds by the fact that $\left|\mathrm{e}^{w}-1\right| \leqslant 2|w|$ for $|w| \leqslant 1$. Thus we obtain

$$
\frac{h^{\prime}(k)}{h(k)} \leqslant \frac{\mu k B}{\sigma^{2}}+\frac{2 \mu k^{2} B^{2}}{\sigma^{3}}
$$

which implies that

$$
\ln h(k) \leqslant \frac{\mu k^{2} B}{2 \sigma^{2}}+\frac{2 \mu k^{3} B^{2}}{3 \sigma^{3}} .
$$

Let $S_{n}=\sum_{i=1}^{n} Y_{i}$ where $Y_{1}, \ldots, Y_{n}$ are nonnegative independent random variables with $E\left(Y_{i}\right)=\mu_{i}$, and let $\mu=E\left(S_{n}\right), \sigma^{2}=\operatorname{Var}\left(S_{n}\right)$ and
$U=\left(S_{n}-\mu\right) / \sigma$. To obtain a non-uniform bound for the independent case, Chen and Shao ${ }^{18}$ used a non-uniform concentration inequality for $U$ by assuming $\left(Y_{i}-\mu_{i}\right) / \sigma \leqslant 1$ for $i=1, \ldots, n$,

$$
P(a \leqslant U \leqslant b) \leqslant \mathrm{e}^{-a / 2}(5(b-a)+7 \gamma)
$$

for any real number $b>a$ where $\gamma=\sum_{i=1}^{n} E \mid\left(Y_{i}-\right.$ $\left.\mu_{i}\right) /\left.\sigma\right|^{3}$. To prove this result, they used the BennettHoeffding inequality

$$
E \mathrm{e}^{t U} \leqslant \exp \left(\mathrm{e}^{t}-1-t\right)
$$

for $t>0{ }^{18}$. They obtained a non-uniform bound of order $\mathrm{e}^{-z}$. To improve the bound to have order $\mathrm{e}^{-z^{2}}$, we follow the proof of a non-uniform concentration inequality for $U$ by choosing a new function $f$ defined by

$$
f(w)= \begin{cases}0, & w<a-\frac{\gamma}{2}, \\ \mathrm{e}^{s w}\left(w-a+\frac{\gamma}{2}\right), & a-\frac{\gamma}{2} \leqslant w \leqslant b+\frac{\gamma}{2}, \\ \mathrm{e}^{s w}(b-a+\gamma), & w>b+\frac{\gamma}{2},\end{cases}
$$

where $s>0$ to obtain

$$
\begin{aligned}
P(a & \leqslant U \leqslant b) \\
& \leqslant 2 \mathrm{e}^{-s a}\left(E \mathrm{e}^{2 s U}\right)^{1 / 2}\left[\mathrm{e}^{s \gamma / 2}(b-a+\gamma)+\frac{\gamma}{2}\right] .
\end{aligned}
$$

In our work, we cannot apply the BennettHoeffding inequality (8) to bound $E \mathrm{e}^{2 s U}$. Thus we use Lemma 1 instead, and then the existence of the size-biased coupling of $S_{n}$ is also needed. For $i=1, \ldots, n$, let $Y_{i}^{s}$ have the $Y_{i}$-size biased distribution, independent of $Y_{j}$ and $Y_{j}^{s}$ for $j \neq i$ and $0 \leqslant Y_{i}^{s}-Y_{i} \leqslant l$ with probability 1 . The size-biased coupling of $S_{n}$ can be constructed from Refs. 22, 24. For a random index $I$ such that

$$
P(I=i)=\frac{\mu_{i}}{\sum_{i=1}^{n} \mu_{i}}
$$

for $i=1, \ldots, n$ and $Y_{1}, \ldots, Y_{n}, I$ are independent, $S_{n}^{s}=S_{n}-Y_{I}+Y_{I}^{s}$ has the $S_{n}$-size biased distribution satisfying

$$
S_{n} \leqslant S_{n}^{s} \leqslant S_{n}+l .
$$

Thus Lemma 1 can be applied with $k=2 s$ and $B=l$ under the assumption that $2 s l \leqslant \sigma$ to obtain

$$
\begin{aligned}
& P(a \leqslant U \leqslant b) \\
& \leqslant 2 \mathrm{e}^{-s a+\mu s^{2} l / \sigma^{2}+8 \mu s^{3} l^{2} / 3 \sigma^{3}}\left[\mathrm{e}^{s \gamma / 2}(b-a+\gamma)+\frac{\gamma}{2}\right] .
\end{aligned}
$$

Hence we obtain the following lemma. 
Lemma 2 Let $S_{n}=\sum_{i=1}^{n} Y_{i}$ where $Y_{1}, \ldots, Y_{n}$ be nonnegative independent random variables with $E\left(Y_{i}\right)=$ $\mu_{i}$ and $Y_{i}^{s}$ having the $Y_{i}$-size biased distribution, independent of $Y_{j}$ and $Y_{j}^{s}$ for $j \neq i$, and let $\mu=E\left(S_{n}\right)$, $\sigma^{2}=\operatorname{Var}\left(S_{n}\right)$ and $U=\left(S_{n}-\mu\right) / \sigma$. For $l>0$, if $0 \leqslant Y_{i}^{s}-Y_{i} \leqslant l$ with probability 1 , for $i=1, \ldots, n$, then for all real numbers $b>a$ and $s>0$ such that $2 s l \leqslant \sigma$,

$$
\begin{aligned}
& P(a \leqslant U \leqslant b) \\
& \leqslant 2 \mathrm{e}^{-s a+\mu s^{2} l / \sigma^{2}+8 \mu s^{3} l^{2} / 3 \sigma^{3}}\left[\mathrm{e}^{s \gamma / 2}(b-a+\gamma)+\frac{\gamma}{2}\right]
\end{aligned}
$$

where $\gamma=\sum_{i=1}^{n} E\left|\left(Y_{i}-\mu_{i}\right) / \sigma\right|^{3}$.

A non-uniform exponential bound is presented in the following theorem.

Theorem 5 Let $S_{n}=\sum_{i=1}^{n} Y_{i}$ where $Y_{1}, \ldots, Y_{n}$ be nonnegative independent random variables with $E\left(Y_{i}\right)=\mu_{i}$ and $Y_{i}^{s}$ having the $Y_{i}$-size biased distribution, independent of $Y_{j}$ and $Y_{j}^{s}$ for $j \neq i$, and let $\mu=E\left(S_{n}\right), \sigma^{2}=\operatorname{Var}\left(S_{n}\right)$ and $U=\left(S_{n}-\mu\right) / \sigma$. Assume that $\left|\left(Y_{i}-\mu_{i}\right) / \sigma\right| \leqslant \delta$ and $0 \leqslant Y_{i}^{s}-Y_{i} \leqslant l$ with probability 1 , for some $l>0$ and for $i=1, \ldots, n$. Then for $z \in \mathbb{R}$ such that $0<\frac{1}{8}|z| l \leqslant \sigma$,

$|P(U \leqslant z)-\Phi(z)| \leqslant 1.5 \delta C(\delta, z+2 \delta)+\frac{2 \delta}{\sqrt{2 \pi}} \mathrm{e}^{-z^{2} / 2}$

where

$$
\begin{aligned}
& C(\delta, z)=\left(\frac{1+z^{2}}{|z|}\right) \mathrm{e}^{-19 z^{2} / 200}+\frac{2 \mathrm{e}^{-z^{2} / 2}}{\sqrt{2 \pi}} \\
& +\left(\frac{1+z^{2}}{|z|}\right) \mathrm{e}^{-9 z^{2} / 80+|z| \delta / 4+\mu z^{2} l / 128 \sigma^{2}+\mu|z|^{3} l^{2} / 768 \sigma^{3}} .
\end{aligned}
$$

Proof: Suppose that $z>0$. For $z<0$, we use the fact that $\Phi(z)=1-\Phi(-z)$ and then apply the result to $-U$. To prove this theorem conveniently, we let

$$
U_{i}=\frac{Y_{i}-\mu_{i}}{\sigma}, \quad U^{(i)}=U-U_{i}
$$

and $K_{i}(t)=E U_{i}\left[\mathbb{I}\left(0 \leqslant t \leqslant U_{i}\right)-\mathbb{I}\left(U_{i} \leqslant t<0\right)\right]$ for $i=1,2, \ldots, n$. Then

$$
\sum_{i=1}^{n} \int_{-\infty}^{\infty} K_{i}(t) \mathrm{d} t=\sum_{i=1}^{n} E U_{i}^{2}=1 .
$$

Since $\left|U_{i}\right| \leqslant \delta, K_{i}(t)=0$ for $|t|>\delta$ implies that

$$
\begin{aligned}
& \sum_{i=1}^{n} \int_{-\infty}^{\infty} P\left(U^{(i)}+t \leqslant z\right) K_{i}(t) \mathrm{d} t \\
& \quad=\sum_{i=1}^{n} \int_{|t| \leqslant \delta} P\left(U-U_{i}+t \leqslant z\right) K_{i}(t) \mathrm{d} t
\end{aligned}
$$

$$
\begin{aligned}
& \geqslant \sum_{i=1}^{n} \int_{|t| \leqslant \delta} P(U \leqslant z-2 \delta) K_{i}(t) \mathrm{d} t \\
& =P(U \leqslant z-2 \delta) \sum_{i=1}^{n} \int_{-\infty}^{\infty} K_{i}(t) \mathrm{d} t \\
& =P(U \leqslant z-2 \delta) .
\end{aligned}
$$

Chen and Shao ${ }^{18}$ proved that

$$
\begin{aligned}
\sum_{i=1}^{n} \int_{-\infty}^{\infty} P\left(U^{(i)}\right. & +t \leqslant z) K_{i}(t) \mathrm{d} t-\Phi(z) \\
= & \sum_{i=1}^{n} E \int_{-\infty}^{\infty}\left\{U f_{z}(U)\right. \\
& \left.-\left(U^{(i)}+t\right) f_{z}\left(U^{(i)}+t\right)\right\} K_{i}(t) \mathrm{d} t .
\end{aligned}
$$

This implies that

$$
\begin{aligned}
\sum_{i=1}^{n} \int_{-\infty}^{\infty} P\left(U^{(i)}+t \leqslant z\right) K_{i}(t) \mathrm{d} t-\Phi(z) \\
\leqslant \sum_{i=1}^{n} E \int_{-\infty}^{\infty} \mid U f_{z}(U) \\
\quad-\left(U^{(i)}+t\right) f_{z}\left(U^{(i)}+t\right) \mid K_{i}(t) \mathrm{d} t \\
=\sum_{i=1}^{n} E \int_{|t| \leqslant \delta} \mid\left(U^{(i)}+U_{i}\right) f_{z}\left(U^{(i)}+U_{i}\right) \\
\quad-\left(U^{(i)}+t\right) f_{z}\left(U^{(i)}+t\right) \mid K_{i}(t) \mathrm{d} t \\
=\sum_{i=1}^{n} E \int_{|t| \leqslant \delta} \mid E^{U_{i}}\left\{\left(U^{(i)}+U_{i}\right) f_{z}\left(U^{(i)}+U_{i}\right)\right\} \\
\quad-E^{U_{i}}\left\{\left(U^{(i)}+t\right) f_{z}\left(U^{(i)}+t\right)\right\} \mid K_{i}(t) \mathrm{d} t .
\end{aligned}
$$

Let $g(w)=\left(w f_{z}(w)\right)^{\prime}$. Chen and Shao ${ }^{17}$ showed that $g(w) \geqslant 0$ for all $w \in \mathbb{R}$ and

$$
g(w)= \begin{cases}\left(\tilde{w}\left(1+w^{2}\right)[1-\Phi(w)]-w\right) \Phi(z), & w>z, \\ \left(\tilde{w}\left(1+w^{2}\right) \Phi(w)+w\right)(1-\Phi(z)), & w<z .\end{cases}
$$

Using the fact that ${ }^{18}$

$$
1-\Phi(z) \leqslant \frac{\mathrm{e}^{-z^{2} / 2}}{\sqrt{2 \pi} z} \quad \text { for } z>0,
$$

we have

$$
g(w) \leqslant 2(1-\Phi(z)) \leqslant \frac{2 \mathrm{e}^{-z^{2} / 2}}{\sqrt{2 \pi} z} \leqslant \frac{\mathrm{e}^{-19 z^{2} / 200}}{z}
$$

for $w \leqslant 0$,

$$
\begin{aligned}
g(w) & \leqslant\left(\sqrt{2 \pi}\left(1+z^{2}\right) \mathrm{e}^{81 z^{2} / 200}+z\right)\left(\frac{\mathrm{e}^{-z^{2} / 2}}{z \sqrt{2 \pi}}\right) \\
& =\frac{1+z^{2}}{z} \mathrm{e}^{-19 z^{2} / 200}+\frac{\mathrm{e}^{-z^{2} / 2}}{\sqrt{2 \pi}}
\end{aligned}
$$


for $0<w \leqslant 9 z / 10$, and

$$
\begin{aligned}
g(w) & \leqslant\left(\sqrt{2 \pi}\left(1+z^{2}\right) \mathrm{e}^{z^{2} / 2}+z\right)\left(\frac{\mathrm{e}^{-z^{2} / 2}}{z \sqrt{2 \pi}}\right) \\
& =\frac{1+z^{2}}{z}+\frac{\mathrm{e}^{-z^{2} / 2}}{\sqrt{2 \pi}}
\end{aligned}
$$

for $9 z / 10<w<z$. For $w \geqslant z$, we know that $g(w) \leqslant$ $2 /\left(1+w^{3}\right)^{17}$. From this fact and (14), we have

$$
g(w) \leqslant \frac{1+z^{2}}{z}+\frac{\mathrm{e}^{-z^{2} / 2}}{\sqrt{2 \pi}} \quad \text { for } w>9 z / 10 .
$$

By (9) and the assumption that $\frac{1}{8} z l \leqslant \sigma$, we can apply Lemma 1 with $B=l$ and $k=\frac{1}{8} z$, and use the fact that $U^{(i)} \leqslant U+\delta$ to show that for any $-\delta \leqslant u \leqslant \delta$,

$$
\begin{aligned}
& P\left(U^{(i)}>\frac{9 z}{10}-u\right) \leqslant \mathrm{e}^{-9 z^{2} / 80+z u / 8} E \mathrm{e}^{(z / 8) U^{(i)}} \\
& \quad \leqslant \mathrm{e}^{-9 z^{2} / 80+z \delta / 8} E \mathrm{e}^{(z / 8)(U+\delta)} \\
& \quad=\mathrm{e}^{-9 z^{2} / 80+z \delta / 4} E \mathrm{e}^{(z / 8) U} \\
& \quad \leqslant \mathrm{e}^{-9 z^{2} / 80+z \delta / 4+\mu z^{2} l / 128 \sigma^{2}+\mu z^{3} l^{2} / 768 \sigma^{3}} .
\end{aligned}
$$

Using (13), (15) and (16), we show that for any $-\delta \leqslant u \leqslant \delta$,

$$
\begin{array}{rl}
E g\left(U^{(i)}+u\right) & \\
=E & g\left(U^{(i)}+u\right) \mathbb{I}\left(U^{(i)}+u \leqslant \frac{9 z}{10}\right) \\
& +E g\left(U^{(i)}+u\right) \mathbb{I}\left(U^{(i)}+u>\frac{9 z}{10}\right) \\
\leqslant & \left(\frac{1+z^{2}}{z} \mathrm{e}^{-19 z^{2} / 200}+\frac{\mathrm{e}^{-z^{2} / 2}}{\sqrt{2 \pi}}\right) \\
& +\left(\frac{1+z^{2}}{z}+\frac{\mathrm{e}^{-z^{2} / 2}}{\sqrt{2 \pi}}\right) P\left(U^{(i)}+u>\frac{9 z}{10}\right) \\
\leqslant & \left(\frac{1+z^{2}}{z}\right) \mathrm{e}^{-19 z^{2} / 200}+\frac{2 \mathrm{e}^{-z^{2} / 2}}{\sqrt{2 \pi}} \\
& +\left(\frac{1+z^{2}}{z}\right) \mathrm{e}^{-9 z^{2} / 80+z \delta / 4+\mu z^{2} l / 128 \sigma^{2}+\mu z^{3} l^{2} / 768 \sigma^{3}} \\
= & : C(\delta, z) .
\end{array}
$$

Hence for any $-\delta \leqslant s, t \leqslant \delta$,

$$
\begin{aligned}
& \left|E\left(U^{(i)}+t\right) f_{z}\left(U^{(i)}+t\right)-E\left(U^{(i)}+s\right) f_{z}\left(U^{(i)}+s\right)\right| \\
& \quad=\left|\int_{s}^{t} E g\left(U^{(i)}+u\right) \mathrm{d} u\right| \\
& \quad \leqslant C(\delta, z)(|s|+|t|) .
\end{aligned}
$$

By (11) and (17), we obtain

$$
\begin{aligned}
& \sum_{i=1}^{n} \int_{-\infty}^{\infty} P\left(U^{(i)}+t \leqslant z\right) K_{i}(t) \mathrm{d} t-\Phi(z) \\
& \quad \leqslant C(\delta, z) \sum_{i=1}^{n} E \int_{|t| \leqslant \delta}\left(\left|U_{i}\right|+|t|\right) K_{i}(t) \mathrm{d} t \\
& \quad=C(\delta, z) \sum_{i=1}^{n}\left\{E\left|U_{i}\right| E U_{i}^{2}+0.5 E\left|U_{i}\right|^{3}\right\} \\
& \quad \leqslant 1.5 \delta C(\delta, z) .
\end{aligned}
$$

From (10) and (18),

$$
\begin{aligned}
& P(U \leqslant z-2 \delta)-\Phi(z-2 \delta) \\
& \leqslant \sum_{i=1}^{n} \int_{-\infty}^{\infty} P\left(U^{(i)}+t \leqslant z\right) K_{i}(t) \mathrm{d} t-\Phi(z-2 \delta) \\
& =\sum_{i=1}^{n} \int_{-\infty}^{\infty} P\left(U^{(i)}+t \leqslant z\right) K_{i}(t) \mathrm{d} t-\Phi(z) \\
& \quad+\Phi(z)-\Phi(z-2 \delta) \\
& \leqslant 1.5 \delta C(\delta, z)+\frac{2 \delta}{\sqrt{2 \pi}} \mathrm{e}^{-(z-2 \delta)^{2} / 2} .
\end{aligned}
$$

This implies that

$$
P(U \leqslant z)-\Phi(z) \leqslant 1.5 \delta C(\delta, z+2 \delta)+\frac{2 \delta}{\sqrt{2 \pi}} \mathrm{e}^{-z^{2} / 2} .
$$

Similarly, the lower bound of $P(U \leqslant z)-\Phi(z)$ can be demonstrated.

Corollary 1 Let $B_{n} \sim \operatorname{Binomial}(n, p)$, where $p \in$ $\left[\frac{1}{8}, \frac{1}{2}\right]$. For all real numbers $z$ such that $0<|z| \leqslant$ $\sqrt{n q / p}$,

$$
\begin{aligned}
\mid P\left(\frac{B_{n}-n p}{\sqrt{n p q}}\right. & \leqslant z)-\Phi(z) \mid \\
& \leqslant 1.5 \delta C(\delta, z+2 \delta)+\frac{2 \delta}{\sqrt{2 \pi}} \mathrm{e}^{-z^{2} / 2},
\end{aligned}
$$

where $q=1-p$,

$$
\begin{aligned}
C(\delta, z) & \leqslant\left(\frac{1+z^{2}}{|z|}\right) \mathrm{e}^{-19 z^{2} / 200}+\frac{2 \mathrm{e}^{-z^{2} / 2}}{\sqrt{2 \pi}} \\
& +\left(\frac{1+z^{2}}{|z|}\right) \mathrm{e}^{|z| \delta / 4-z^{2}(9 / 80-1 / 128 q-1 / 768 p q)}
\end{aligned}
$$

and $\delta=\max \{p, q\} / \sqrt{n p q}$. Furthermore, if $p=\frac{1}{2}$, then for all real numbers $z$ such that $|z| \leqslant \sqrt{n}$,

$$
\begin{aligned}
& \left|P\left(\frac{B_{n}-n / 2}{\sqrt{n / 4}} \leqslant z\right)-\Phi(z)\right| \\
& \quad \leqslant \frac{1}{\sqrt{n}}\left[9.6414 \mathrm{e}^{-z^{2} / 2}+21.9638 \mathrm{e}^{-z^{2} / 12}\right] .
\end{aligned}
$$


Proof: Note that $\mu=E\left(B_{n}\right)=n p$ and $\sigma^{2}=$ $\operatorname{Var}\left(B_{n}\right)=n p q$. Let $B_{n}=\sum_{i=1}^{n} Y_{i}$ where $Y_{i} \sim \operatorname{Ber}(p)$. Ross $^{24}$ showed that $Y_{i}^{s}=1$ for $i=1, \ldots, n$. Because $p \geqslant \frac{1}{8}$ and $0<|z| \leqslant \sqrt{n q / p}$, we obtain $0<\frac{1}{8}|z| \leqslant \sigma$. Thus we can apply Theorem 5 with $l=1$ to obtain

$$
\begin{aligned}
\mid P\left(\frac{B_{n}-n p}{\sqrt{n p q}}\right. & \leqslant z)-\Phi(z) \mid \\
& \leqslant 1.5 \delta C(\delta, z+2 \delta)+\frac{2 \delta}{\sqrt{2 \pi}} \mathrm{e}^{-z^{2} / 2},
\end{aligned}
$$

where

$$
\begin{aligned}
& C(\delta, z)=\left(\frac{1+z^{2}}{|z|}\right) \mathrm{e}^{-19 z^{2} / 200}+\frac{2 \mathrm{e}^{-z^{2} / 2}}{\sqrt{2 \pi}} \\
& +\left(\frac{1+z^{2}}{|z|}\right) \mathrm{e}^{-9 z^{2} / 80+|z| \delta / 4+z^{2} / 128 q+|z|^{3} / 768 q \sqrt{n p q}} \\
& \leqslant\left(\frac{1+z^{2}}{|z|}\right) \mathrm{e}^{-19 z^{2} / 200}+\frac{2 \mathrm{e}^{-z^{2} / 2}}{\sqrt{2 \pi}} \\
& +\left(\frac{1+z^{2}}{|z|}\right) \mathrm{e}^{|z| \delta / 4-z^{2}(9 / 80-1 / 128 q-1 / 768 p q)}
\end{aligned}
$$

Consider $p=\frac{1}{2}$. If $|z| \leqslant 4$, by (4), we obtain

$$
\left|P\left(\frac{B_{n}-n / 2}{\sqrt{n / 4}} \leqslant z\right)-\Phi(z)\right| \leqslant 3.3 \delta \leqslant \frac{12.52}{\sqrt{n}} \mathrm{e}^{-z^{2} / 12} .
$$

From (19), if $|z|>4$, by using the property that $|z| \leqslant$ $\sqrt{n}$ and $\delta=1 / \sqrt{n}$, we have

$$
\begin{aligned}
C(\delta, z) \leqslant & \left(\frac{1+z^{2}}{|z|}\right) \mathrm{e}^{-19 z^{2} / 200}+\frac{2 \mathrm{e}^{-z^{2} / 2}}{\sqrt{2 \pi}} \\
& +\left(\frac{1+z^{2}}{|z|}\right) \mathrm{e}^{1 / 4-z^{2}(9 / 80-1 / 64-1 / 192)} \\
= & \left.\frac{1+z^{2}}{|z|}\right) \mathrm{e}^{-19 z^{2} / 200}+\frac{2 \mathrm{e}^{-z^{2} / 2}}{\sqrt{2 \pi}} \\
& +\left(\frac{1+z^{2}}{|z|}\right) \mathrm{e}^{1 / 4-88 z^{2} / 960} \\
= & \left.\frac{1+z^{2}}{|z|}\right) \mathrm{e}^{-7 z^{2} / 600-z^{2} / 12}+\frac{2 \mathrm{e}^{-z^{2} / 2}}{\sqrt{2 \pi}} \\
& +\left(\frac{1+z^{2}}{|z|}\right) \mathrm{e}^{1 / 4-z^{2} / 120-z^{2} / 12} \\
\leqslant & \frac{2 \mathrm{e}^{-z^{2} / 2}}{\sqrt{2 \pi}}+10.4918 \mathrm{e}^{-z^{2} / 12}
\end{aligned}
$$

where the last inequality holds by using the fact that $\mathrm{e}^{-7 z^{2} / 600} /|z| \leqslant 0.2075,|z| \mathrm{e}^{-7 z^{2} / 600} \leqslant 3.9707$, $\mathrm{e}^{-z^{2} / 120} /|z| \leqslant 0.2188$ and $|z| \mathrm{e}^{-z^{2} / 120} \leqslant 4.6982$ for $|z| \geqslant 4$. This implies that

$$
\begin{aligned}
C(\delta, z+2 \delta) & \leqslant \frac{2 \mathrm{e}^{-(z+2 \delta)^{2} / 2}}{\sqrt{2 \pi}}+10.4918 \mathrm{e}^{-(z+2 \delta)^{2} / 12} \\
& \leqslant \frac{2 \mathrm{e}^{-z^{2} / 2+2|z| \delta}}{\sqrt{2 \pi}}+10.4918 \mathrm{e}^{|z| \delta / 3-z^{2} / 12} \\
& \leqslant \frac{2 \mathrm{e}^{2} \mathrm{e}^{-z^{2} / 2}}{\sqrt{2 \pi}}+10.4918 \mathrm{e}^{1 / 3-z^{2} / 12} .
\end{aligned}
$$

Thus for all $z \leqslant \sqrt{n}$,

$$
\begin{aligned}
& \left|P\left(\frac{B_{n}-n / 2}{\sqrt{n / 4}} \leqslant z\right)-\Phi(z)\right| \\
& \quad \leqslant 1.5 \delta C(\delta, z+2 \delta)+\frac{2 \delta}{\sqrt{2 \pi}} \mathrm{e}^{-z^{2} / 2} \\
& \quad \leqslant \frac{1}{\sqrt{n}}\left[9.6414 \mathrm{e}^{-z^{2} / 2}+21.9638 \mathrm{e}^{-z^{2} / 12}\right] .
\end{aligned}
$$

\section{PROOF OF THEOREM 3}

It suffices to prove the theorem in the case $z>0$. Let $B_{n-1} \sim \operatorname{Binomial}\left(n-1, \frac{1}{2}\right)$. Note that

$$
\begin{gathered}
\left|P\left(\frac{W_{n}-n / 2}{\sigma} \leqslant z\right)-\Phi(z)\right| \\
\leqslant\left|P\left(\frac{W_{n}-n / 2}{\sigma} \leqslant z\right)-P\left(\frac{C_{n}-n / 2}{\sigma} \leqslant z\right)\right| \\
+\left|P\left(\frac{C_{n}-n / 2}{\sigma} \leqslant z\right)-P\left(\frac{B_{n-1}-n / 2}{\sigma} \leqslant z\right)\right| \\
+\mid P\left(\frac{B_{n-1}-n / 2}{\sigma} \leqslant z\right) \\
\quad-P\left(\frac{B_{n-1}-(n-1) / 2}{\sqrt{(n-1) / 4}} \leqslant z\right) \mid \\
+\left|P\left(\frac{B_{n-1}-(n-1) / 2}{\sqrt{(n-1) / 4}} \leqslant z\right)-\Phi(z)\right| \\
=: A_{1}+A_{2}+A_{3}+A_{4} .
\end{gathered}
$$

By (3) and $z \leqslant \sqrt{n}$, we see that

$$
\begin{aligned}
A_{1} \leqslant 2.7314 \sqrt{n} \mathrm{e}^{-(n+1) / 3} & \\
\leqslant & 2.7314 \sqrt{n} \mathrm{e}^{-(n+1) / 12-\left(z^{2}+1\right) / 4} .
\end{aligned}
$$

Since $n$ is even, $n \bmod 4 \in\{0,2\}$. Suppose that $n \bmod 4=0$. Let $t$ be any real number such that $t \in(0, n]$. If $\lfloor t\rfloor$ is even, then

$$
\begin{aligned}
& \left|P\left(C_{n} \leqslant\lfloor t\rfloor\right)-P\left(B_{n-1} \leqslant\lfloor t\rfloor\right)\right| \\
& \quad=\left|\sum_{i=0}^{\lfloor t\rfloor} P\left(C_{n}=i\right)-\sum_{i=0}^{\lfloor t\rfloor} P\left(B_{n-1}=i\right)\right|
\end{aligned}
$$




$$
\begin{aligned}
& =\left(\frac{1}{2}\right)^{n-1}\left|\sum_{i=0}^{\lfloor t\rfloor / 2}\left(\begin{array}{c}
n \\
2 i
\end{array}\right)-\sum_{i=0}^{\lfloor t\rfloor}\left(\begin{array}{c}
n-1 \\
i
\end{array}\right)\right| \\
& =\left(\frac{1}{2}\right)^{n-1}\left|\sum_{i=1}^{\lfloor t\rfloor / 2}\left[\left(\begin{array}{c}
n \\
2 i
\end{array}\right)-\left(\begin{array}{c}
n-1 \\
2 i
\end{array}\right)\right]-\sum_{i=1}^{\lfloor t\rfloor / 2}\left(\begin{array}{c}
n-1 \\
2 i-1
\end{array}\right)\right| \\
& =0
\end{aligned}
$$

where the last equality is true by using the property that $\left(\begin{array}{l}n \\ k\end{array}\right)-\left(\begin{array}{c}n-1 \\ k\end{array}\right)=\left(\begin{array}{l}n-1 \\ k-1\end{array}\right)$ for all integers $n, k$ such that $1 \leqslant k \leqslant n-1$. If $\lfloor t\rfloor$ is odd, then for $\lfloor t\rfloor=1$,

$$
\begin{aligned}
& \left|P\left(C_{n} \leqslant\lfloor t\rfloor\right)-P\left(B_{n-1} \leqslant\lfloor t\rfloor\right)\right| \\
& =\left|P\left(C_{n}=0\right)-\sum_{i=0}^{1} P\left(B_{n-1}=i\right)\right|=\left(\frac{1}{2}\right)^{n-1}\left(\begin{array}{c}
n-1 \\
1
\end{array}\right)
\end{aligned}
$$

and for $\lfloor t\rfloor \geqslant 3$, we have

$$
\begin{aligned}
& \left|P\left(C_{n} \leqslant\lfloor t\rfloor\right)-P\left(B_{n-1} \leqslant\lfloor t\rfloor\right)\right| \\
& =\left|\sum_{i=0}^{\lfloor t\rfloor-1} P\left(C_{n}=i\right)-\sum_{i=0}^{\lfloor t\rfloor} P\left(B_{n-1}=i\right)\right| \\
& =\left(\frac{1}{2}\right)^{n-1}\left|\sum_{i=0}^{\mid\lfloor t\rfloor-1) / 2}\left(\begin{array}{c}
n \\
2 i
\end{array}\right)-\sum_{i=0}^{\lfloor t\rfloor}\left(\begin{array}{c}
n-1 \\
i
\end{array}\right)\right| \\
& =\left(\frac{1}{2}\right)^{n-1} \mid \sum_{i=1}^{(\lfloor t\rfloor-1) / 2}\left[\left(\begin{array}{c}
n \\
2 i
\end{array}\right)-\left(\begin{array}{c}
n-1 \\
2 i
\end{array}\right)\right\rfloor \\
& \quad-\sum_{i=1}^{(\lfloor t) / 2}\left(\begin{array}{c}
n-1 \\
2 i-1
\end{array}\right)-\left(\begin{array}{c}
n-1 \\
\lfloor t\rfloor
\end{array}\right) \mid \\
& =\left(\frac{1}{2}\right)^{n-1}\left(\begin{array}{c}
n-1 \\
\lfloor t\rfloor
\end{array}\right) .
\end{aligned}
$$

In the case that $n \bmod 4=2$, we use the same argument to obtain

$$
\begin{aligned}
\mid P\left(C_{n} \leqslant\lfloor t\rfloor\right) & -P\left(B_{n-1} \leqslant\lfloor t\rfloor\right) \mid \\
= & \begin{cases}\left(\begin{array}{c}
n-1 \\
\lfloor t\rfloor
\end{array}\right)\left(\frac{1}{2}\right)^{n-1}, & \lfloor t\rfloor \text { is even, } \\
0, & \lfloor t\rfloor \text { is odd. }\end{cases}
\end{aligned}
$$

Hence

$$
\begin{aligned}
A_{2} & =\left|P\left(C_{n} \leqslant\left\lfloor\sigma z+\frac{n}{2}\right\rfloor\right)-P\left(B_{n-1} \leqslant\left\lfloor\sigma z+\frac{n}{2}\right\rfloor\right)\right| \\
& \leqslant P\left(B_{n-1}=\left\lfloor\sigma z+\frac{n}{2}\right\rfloor\right) .
\end{aligned}
$$

Next, consider $A_{3}$. By (1) and the fact that $\mathrm{e}^{n} \geqslant n$ for any $n \in \mathbb{N}$, we see that

$$
\sqrt{\frac{n-1}{4}} \leqslant \sqrt{\frac{n}{4}\left(1-\mathrm{e}^{-n}\right)} \leqslant \sigma
$$

and for $z \leqslant \sqrt{n}$,

$$
\begin{aligned}
& \left(\sigma-\sqrt{\frac{n-1}{4}}\right) z \\
& \leqslant \frac{n \mathrm{e}^{-n}+1}{\sqrt{4 n\left(1+\mathrm{e}^{-n}\right)}+\sqrt{4(n-1)}} z \\
& \leqslant \frac{1}{2} \frac{n \mathrm{e}^{-n}+1}{\sqrt{1+\mathrm{e}^{-n}}+\sqrt{1-1 / n}}<\frac{1}{2}
\end{aligned}
$$

for $n \geqslant 6$. Hence

$$
\frac{1}{2} \leqslant\left(\sigma z+\frac{n}{2}\right)-\left(\sqrt{\frac{n-1}{4}} z+\frac{n-1}{2}\right)<1
$$

which implies

$$
\begin{aligned}
A_{3} & =P\left(\sqrt{\frac{n-1}{4}} z+\frac{n-1}{2}<B_{n-1} \leqslant \sigma z+\frac{n}{2}\right) \\
& \leqslant P\left(B_{n-1}=\left\lfloor\sigma z+\frac{n}{2}\right\rfloor\right) .
\end{aligned}
$$

We now use the fact that $x-1 \leqslant\lfloor x\rfloor \leqslant x$ for any $x \in \mathbb{R}$ and $\frac{1}{5} z \leqslant \sqrt{(n-1) / 4}$, and then we can apply Lemma 2 to $\left(B_{n-1}-(n-1) / 2\right) / \sqrt{(n-1) / 4}$ equipped with $l=1, s=\frac{1}{10} z, \gamma=1 /(\sqrt{n-1})$ and $\sigma^{2} \geqslant 0.249 n$ for $n \geqslant 6$ to obtain

$$
\begin{aligned}
A_{2}+A_{3} & \\
\leqslant 2 P & \left(\sigma z+\frac{n}{2}-1 \leqslant B_{n-1} \leqslant \sigma z+\frac{n}{2}\right) \\
=2 P( & \frac{2 \sigma}{\sqrt{n-1}} z-\frac{1}{\sqrt{n-1}} \leqslant \frac{B_{n-1}-(n-1) / 2}{\sqrt{(n-1) / 4}} \\
& \left.\leqslant \frac{2 \sigma}{\sqrt{n-1}} z+\frac{1}{\sqrt{n-1}}\right) \\
\leqslant 4 & \mathrm{e}^{-(z / 10)((2 \sigma /(\sqrt{n-1})) z-1 /(\sqrt{n-1}))+(351 / 10000) z^{2}} \\
& \times\left[\mathrm{e}^{\sqrt{2} / 20}\left(\frac{3}{\sqrt{n-1}}\right)+\frac{1}{2 \sqrt{n-1}}\right] \\
\leqslant 4 & \mathrm{e}^{-(\sigma /(5 \sqrt{n-1})) z^{2}+\sqrt{2} / 10+(351 / 10000) z^{2}} \\
& \times\left[\mathrm{e}^{\sqrt{2} / 20}\left(\frac{3}{\sqrt{n-1}}\right)+\frac{1}{2 \sqrt{n-1}}\right] \\
\leqslant & 4 \mathrm{e}^{-(997 / 10000) z^{2}+\sqrt{2} / 10+(351 / 10000) z^{2}} \\
& \times\left[\mathrm{e}^{\sqrt{2} / 20}\left(\frac{3}{\sqrt{n-1}}\right)+\frac{1}{2 \sqrt{n-1}}\right] \\
\leqslant & \frac{17.1396}{\sqrt{n-1}} \mathrm{e}^{-z^{2} / 16} \cdot
\end{aligned}
$$

It remains to bound the error term $A_{4}$. Applying Corollary 1 to $B_{n-1} \sim \operatorname{Binomial}\left(n-1, \frac{1}{2}\right)$, we obtain

$$
A_{4} \leqslant \frac{1}{\sqrt{n-1}}\left[9.6414 \mathrm{e}^{-z^{2} / 2}+21.9638 \mathrm{e}^{-z^{2} / 12}\right] \text {. }
$$


Hence, by (20)-(23), the theorem holds.

\section{PROOF OF THEOREM 4}

Note that

$$
\begin{array}{r}
\left|P\left(\frac{W_{n}-n / 2}{\sigma_{V}} \leqslant z\right)-\Phi(z)\right| \\
\leqslant\left|P\left(\frac{W_{n}-n / 2}{\sigma_{V}} \leqslant z\right)-P\left(\frac{C_{n}-n / 2}{\sigma_{V}} \leqslant z\right)\right| \\
+\left|P\left(\frac{C_{n}-n / 2}{\sigma_{V}} \leqslant z\right)-P\left(\frac{B_{n-1}-n / 2}{\sigma_{V}} \leqslant z\right)\right| \\
+\mid P\left(\frac{B_{n-1}-n / 2}{\sigma_{V}} \leqslant z\right) \\
-P\left(\frac{B_{n-1}-(n-1) / 2}{\sqrt{(n-1) / 4}} \leqslant z\right) \mid \\
+\left|P\left(\frac{B_{n-1}-(n-1) / 2}{\sqrt{(n-1) / 4}} \leqslant z\right)-\Phi(z)\right| .
\end{array}
$$

Using the same argument as in Theorem 3, we can show that

$$
\left|P\left(\frac{W_{n}-n / 2}{\sigma_{V}} \leqslant z\right)-\Phi(z)\right| \leqslant \varepsilon(n, z)
$$

as desired.

Acknowledgements: The authors are very thankful for the useful comments from the referees and financial support by the Science Achievement Scholarship of Thailand.

\section{REFERENCES}

1. Clough V, Yoshida K, Nakaza M, Guidotti TL (1995) Field performance of dermal patches in pesticide exposure determinations. In: Dosman JA, McDuffie HH, Olenchock S, Semchuk K, Senthilselvan A (eds) Agricultural Health and Safety Workplace, Environment, Sustainability, CRC Press, pp 241-5.

2. Houghton D, Archibald BA, Solomon KR (1998) Review of video imaging techniques for estimating dermal exposure to pesticides. In: Ecobichon DJ (ed) Occupational Hazards of Pesticide Exposure: Sampling, Monitoring, Measuring, Taylor \& Francis, pp 135-86.

3. Garino JP, Beredjiklian PK (2007) Adult Reconstruction and Arthroplasty, Elsevier Health Sciences.

4. Rao CR, Rao MB, Zhang H (2007) One bulb? Two bulbs? How many bulbs light up?-a discrete probability problem involving dermal patches. Sankhya 69 , 137-61.

5. Sharma N, Parashar B, Sharma S, Mahajan U (2012) Blooming pharma industry with transdermal drug delivery system. Indo Global J Pharmaceut Sci 2, 262-78.
6. Goldstein L, Zhang H (2011) A Berry-Esseen bound for the lightbulb process. Adv Appl Probab 43, 875-98.

7. Goldstein L, Xia A (2012) Clubbed binomial approximation for the lightbulb process. In: Barbour AD, Chan HP, Siegmund D (eds) Probability Approximations and Beyond, Springer, New York, pp 31-41.

8. Esseen CG (1942) On the Liapounoff limit of error in the theory of probability. Ark Mat Astron Fys 28A, $1-19$.

9. Nagaev SV (1965) Some limit theorems for large deviations. Theor Probab Appl 10, 214-35.

10. Korolev VY, Shevtsova IG (2010) On the upper bound for the absolute constant in the Berry-Esseen inequality. Theor Probab Appl 54, 638-58.

11. Shiganov IS (1986) Refinement of the upper bound of the constant in the central limit theorem. $J$ Sov Math 35, 2545-50.

12. Tyurin I (2009) New estimates of the convergence rate in the Lyapunov theorem. arXiv:0912.0726.

13. Shevtsova I (2011) On the absolute constants in the Berry-Esseen type inequalities for identically distributed summands. arXiv:1111.6554.

14. Bikelis A (1966) Estimates of the remainder in a combinatorial central limit theorem. Litovsk Math $\mathrm{Sb}$ 6, 323-46.

15. Shevtsova IG (2010) An improvement of convergence rate estimates in the Lyapunov theorem. Dokl Math 82, 862-4.

16. Paditz L (1989) On the analytical structure of the constant in the nonuniform version of the Esseen inequality. Statistics 20, 453-64.

17. Chen LHY, Shao QM (2001) A non-uniform BerryEsseen bound via Stein's method. Probab Theor Relat Field 120, 236-54.

18. Chen LHY, Shao QM (2005) Stein's method for normal approximation. In: Barbour AD, Chen LHY (eds) An Introduction to Stein's Method, Lecture Notes Series, Institute for Mathematical Sciences, National Univ of Singapore, vol 4, Singapore Univ Press, pp 1-59.

19. Chaidee N (2005) Non-uniform bounds in normal approximation for matrix correlation statistics and independent bounded random variables. $\mathrm{PhD}$ thesis, Chulalongkorn Univ.

20. Chen LHY, Fang X, Shao QM (2013) From Stein identities to moderate deviations. Ann Probab 41, 262-93.

21. Stein C (1972) A bound for the error in normal approximation to the distribution of a sum of dependent random variables. In: Proceedings of the Sixth Berkeley Symposium on Mathematical Statistics and Probability, vol 2, Univ of California Press, pp 583-602.

22. Chen LHY, Goldstein L, Shao QM (2011) Normal Approximation by Stein's Method, Springer, New York.

23. Goldstein L (2005) Berry-Esseen bounds for combi- 
natorial central limit theorems and pattern occurrences, using zero and size biasing. J Appl Probab 42, 661-83.

24. Ross N (2011) Fundamentals of Stein's method. Probab Surv 8, 210-93. 